\title{
Increased Frequency of Heterozygous Alpha-1-Antitrypsin Deficiency in Liver Explants From Nonalcoholic Steatohepatitis Patients
}

\author{
Gregory Cheeney, ${ }^{1,2}$ Lincoln J. Pac, ${ }^{3}$ Purva Gopal, ${ }^{4}$ Charles S. Landis, ${ }^{5}$ Eric Q. Konnick, ${ }^{2}$ \\ Paul E. Swanson, ${ }^{1}$ Dina N. Greene, ${ }^{2}$ Christina M. Lockwood, ${ }^{2}$ and Maria Westerhoff ${ }^{6}$ \\ Departments of ${ }^{1}$ Pathology and ${ }^{2}$ Department of Laboratory Medicine, University of Washington School of Medicine, Seattle, WA; \\ ${ }^{3}$ Department of Pathology, University of Utah School of Medicine, Salt Lake City, UT; ${ }^{4}$ Department of Pathology, University of Texas \\ Southwestern Medical Center, Dallas, TX; ${ }^{5}$ Department of Medicine, University of Washington School of Medicine, Seattle, WA; and \\ ${ }^{6}$ Department of Pathology, University of Michigan Health System, Ann Arbor, MI
}

\begin{abstract}
Cirrhotic explanted livers occasionally have unexpected periodic acid-Schiff-diastase (PASD)-positive globules within the hepatocyte cytoplasm. It is often unclear whether this finding is a nonspecific consequence of cirrhosis or is indicative of an underlying alpha-1-antitrypsin deficiency (A1ATD) contributing to the cirrhosis. In this study, explanted livers were retrospectively evaluated for histopathology (including PASD status with confirmatory alpha-1-antitrypsin [A1AT] immunohistochemistry [IHC]), and chart review provided etiology of liver failure and general clinical parameters. Real-time polymerase chain reaction was used to detect A1AT genotype (SERPINA1 S and $\mathrm{Z}$ alleles) by melting curve analysis on liver explant tissue from selected cases. Of 196 explanted livers, 21 (11\%) had PASD+ globules, which were significantly enriched in patients with a clinical diagnosis of nonalcoholic steatohepatitis (NASH; 47\%) compared with other causes $(P<0.001)$. IHC confirmed all PASD+ globules were A1AT +, with 20 of 21 cases demonstrating diffuse A1AT staining. In an expanded NASH cohort, $42 \%$ $(14 / 33)$ of explants had PASD+ globules, $92 \%$ of which were homozygous $(\mathrm{n}=1)$ or heterozygous $(\mathrm{n}=11)$ for the SERPINA1 $\mathrm{Z}$ allele, corresponding to nearly $40 \%$ of all NASH patients. Overall, the $\mathrm{Z}$ allele was present in $10 \%$ of all tested liver explants, with $85 \%$ of PASD + cases genotyping homozygous $(n=2)$ or heterozygous $(n=20)$, which is far in excess of the estimated $2 \%$ in the general population. These results indicate PASD+A1AT globules (with confirmatory genotyping showing at least $1 \mathrm{Z}$ allele) are commonly observed in NASH, suggesting a synergistic relationship toward liver fibrosis. In addition, the high frequency of SERPINA1 $\mathrm{Z}$ alleles in liver transplantation patients supports the utility of pretransplant genotyping.
\end{abstract}

Liver Transplantation 26 17-24 2020 AASLD.

Received May 2, 2019; accepted August 15, 2019.

Alpha-1-antitrypsin deficiency (A1ATD) is an autosomal recessive genetic disease with incomplete penetrance that can lead to progressive pulmonary and liver disease. The diagnosis of A1ATD is most commonly established by the demonstration of low serum

Abbreviations: A1AT, alpha-1-antitrypsin; A1ATD, alpha1-antitrypsin deficiency; BMI, body mass index; Ct, threshold cycle; ESLD, end-stage liver disease; EtOH, alcohol; FFPE, formalin-fixed paraffin-embedded; HCV, hepatitis $C$ virus; IHC, immunohistochemistry; NASH, nonalcoholic steatohepatitis; PASD, periodic acid-Schiff-diastase; $Q C$, quality control; RT-PCR, real-time polymerase chain reaction; $S D$, standard deviation. alpha-1-antitrypsin (A1AT) concentration and/or isoelectric focusing of serum proteins. ${ }^{(1)}$ A1AT serum deficiency is caused by mutations in the SERPINA1 gene, leading to protein misfolding or transcript instability that is sometimes accompanied by A1AT polymerization within hepatocytes. ${ }^{(2)}$ The SERPINA1 $\mathrm{S}$ and Z (NM_000295.4: c.863A>T, p.E288V and c.1096G >A, p.E366K, respectively) alleles account for $95 \%$ of A1AT deficiency alleles. ${ }^{(1,2)}$ If polymerization occurs, the A1AT glycoprotein accumulates in the endoplasmic reticulum of hepatocytes to form cytoplasmic globules that may be visualized on routine histology and highlighted by periodic acid-Schiffdiastase (PASD) stain. This accumulated protein 
is thought to be the primary cause of liver injury in A1ATD. ${ }^{(3)}$

Liver transplantation is the last treatment option for patients suffering from end-stage liver disease (ESLD) due to cirrhosis. Liver explants removed from patients with ESLD due to cirrhosis may contain PASD+ cytoplasmic globules within hepatocytes. These globules have been previously divided into type 1 and type 2 globules. Type 1 globules are located in the periportal region and are associated with cirrhosis. Type 2 globules are in a centrilobular region and are generally not associated with cirrhosis. A1AT globules in A1ATD are characteristically type 1 PASD globules. ${ }^{(4)}$ The PASD stain has been shown to be a good screening stain to identify hepatocyte A1AT globules; however, A1AT immunohistochemical staining is still useful to help confirm that the globule is A1AT. ${ }^{(5)}$ Type 1 PASD+ globules may be unexpectedly encountered in liver explants that were not performed for A1ATD. In fact, in the majority of cirrhotic livers with incidentally discovered PASD+ globules, there is no documented history of A1ATD. ${ }^{(6)}$ Prior studies estimate that diffuse cirrhotic type PASD+ globules occur in approximately $10 \%$ of patients with $\mathrm{ESLD},{ }^{(6)}$ an observation that raises concerns about the specificity of type 1 PASD + globules in the setting of liver explanation.

The contribution of A1ATD to the development of ESLD, particularly MZ heterozygosity, is an evolving area of research. ${ }^{(7-11)}$ Multiple studies have identified increased rates of $\mathrm{Z}$ allele heterozygosity in advanced liver disease ${ }^{(10,12)}$; however, not all studies have shown consistent results. ${ }^{(13)}$ Furthermore, the majority of liver transplants performed for documented A1ATD ( 88\%) occur in older adults (peak age range of 50-64 years), leading some authors to speculate that the principle phenotype of A1ATD

Address reprint requests to Maria Westerhoff, M.D., Department of Pathology, University of Michigan Health System, University Hospital Floor 2, 1500 East Medical Center Drive, SPC 5054, Ann Arbor, MI 48109. Telephone: 800-862-7284; FAX: 734-615-2964; E-mail: mariawesterhoff@gmail.com

Charles S. Landis has grants from Gilead, HighTide Therapeutics, and Allergan. Paul E. Swanson is employed by the College of American Pathologists.

Copyright (C) 2019 by the American Association for the Study of Liver Diseases.

View this article online at wileyonlinelibrary.com.

DOI 10.1002/lt.25652 may be an age-dependent degenerative disease. ${ }^{(14)}$ However, it is unclear how comorbidities, such as nonalcoholic steatohepatitis (NASH), might play a role in an age-dependent degenerative disease model of A1ATD.

Individuals with unexplained chronic liver disease should be tested for A1ATD, and those found to be positive for a mutant A1ATD allele should be followed with annual imaging and laboratory evaluations. Family members of affected individuals should be provided with genetic counseling. ${ }^{(15)}$ The significance and management of unexpected PASD+ globules in adult liver explants is less clear. The identification of A1AT globules in the explanted liver may not be a priority for clinical management because the transplant is curative. The ramifications on family members, however, should be considered, regardless of whether the patient is cured. In this study, we evaluated the frequency of PASD+ globules in liver explants with A1AT immunohistochemical confirmation, noted patient characteristics, and assessed the presence of the SERPINA1 gene $\mathrm{Z}$ and $\mathrm{S}$ alleles using a novel genetic assay developed and validated within our clinical laboratory. ${ }^{(16)}$ The study was approved by the institutional review boards of the respective institutions.

\section{Patients and Methods}

Cirrhotic livers removed at the time of orthotopic transplantation for ESLD during a 3-year period (January 1, 2013 to December 31, 2015) from a single institution were retrospectively reviewed after full institutional review board approval. No organs from executed prisoners were used in this study. For each case, liver pathology, including the degree of cirrhosis, presence of cytoplasmic globules within hepatocytes, and positivity of the globules on PASD staining, were reviewed by 2 pathologists (M.W. and G.C.). PASD staining was performed on an automated platform (Artisan Link; Dako, Carpinteria, CA). All cases were stained with PASD, and all cases positive for PASD globules had confirmatory A1AT immunohistochemistry (IHC) performed. The latter immunostaining was performed on an automated platform (Bond III; Leica, Buffalo Grove, IL) using a polyclonal anti-A1AT antibody (1:750, Cell Marque, Rocklin, CA) and developed with a polymer-based detection kit (Bond Polymer Refine; Leica) for chromogen development on the slide. A readout of the A1AT immunohistochemical assays was performed by 2 pathologists (M.W. and G.C.) who were blinded to the histologic and clinical diagnosis. 
General clinical parameters, including age, sex, etiology of ESLD, and body mass index (BMI) at time of transplantation, were extracted from retrospective chart review for each patient. Initial diagnostic liver biopsies were generally unavailable for evaluation; therefore, histopathologic features of these biopsies were not included in the demographic data set. Serum A1AT concentrations from any time point in the patient's care were collected if available.

In a second round of case reviews following initial observations of the described cohort, all liver explants with NASH as the underlying etiology from 2006 to 2012 (from the same institution as the primary explant cohort) were similarly assessed. A1AT IHC was performed retrospectively on all NASH cases, both PASD+ and PASD-. We used the Student $t$ test to compare means of continuous variables, and we used Fisher's exact test to compare categorical data. A $P$ value $<0.05$ was considered significant.

To determine the A1AT genotype of the archived samples in this retrospective analysis, our group validated a real-time polymerase chain reaction (RT-PCR) method using formalin-fixed paraffin-embedded (FFPE) liver tissue. ${ }^{(16)}$ Briefly, genomic DNA was extracted from FFPE blocks of liver tissue from all PASD+ cases and all PASD- NASH cases. RT-PCR was employed to amplify SERPINA1 to detect the S and $Z$ alleles (NM_000295.4: c.863A $>$ T, p.E288V and c.1096G >A, p.E366K, respectively) using allele-specific fluorescence resonance energy transfer probes and melting curve analysis. Validation samples had mean melting temperatures for the $Z\left(61.2^{\circ} \mathrm{C}\right.$; standard deviation $\left.[\mathrm{SD}]=0.34^{\circ} \mathrm{C}\right)$ and $\mathrm{S}\left(55.4^{\circ} \mathrm{C} ; \mathrm{SD}=0.30^{\circ} \mathrm{C}\right)$ alleles that were clearly separated from the non $Z\left(54.7^{\circ} \mathrm{C}\right.$; $\left.\mathrm{SD}=0.19^{\circ} \mathrm{C}\right)$ and non $-\mathrm{S}\left(48.6^{\circ} \mathrm{C} ; \mathrm{SD}=0.28^{\circ} \mathrm{C}\right)$ alleles.

\section{Results}

During the 2013-2015 study period, 196 (male:female, $\mathrm{n}=133: 63$ ) liver explants were removed at our institution during liver transplantation for ESLD. The etiologies of ESLD in the liver explants included hepatitis $\mathrm{C}$ virus (HCV)-induced cirrhosis $(\mathrm{n}=90)$; chronic alcohol use-related cirrhosis $(n=43)$; NASH-related cirrhosis $(n=15)$; and a mixture of other diagnoses including (among others) primary sclerosing cholangitis and autoimmune hepatitis ( $n=61$; Fig. 1 ). A subset of patients $(n=13)$ had 2 etiologies clinically considered to contribute to ESLD.

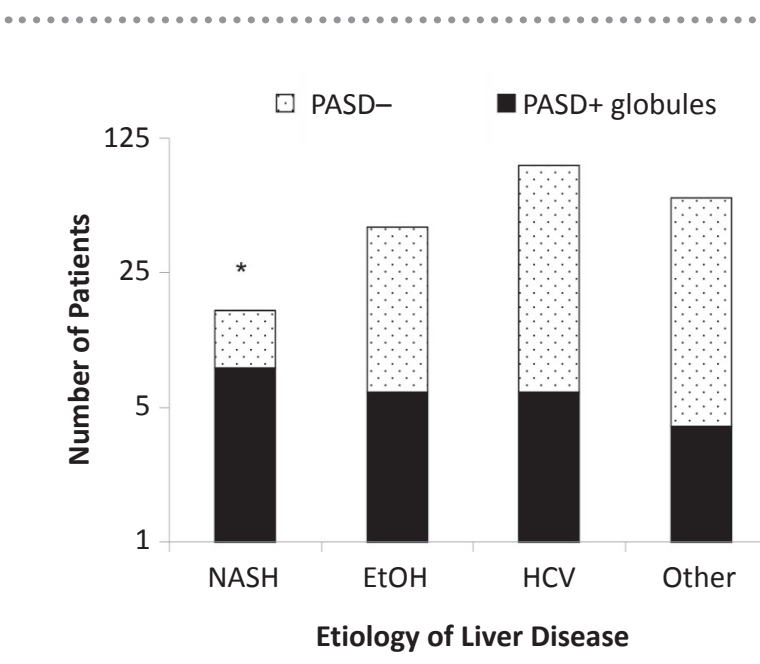

FIG. 1. The etiologies of ESLD in the liver explants were as follows: 90 patients with $\mathrm{HCV}$-induced cirrhosis; 43 patients with chronic alcohol use-related cirrhosis $(\mathrm{EtOH}) ; 15$ patients with NASH-related cirrhosis; and 61 patients with a mixture of other diagnoses including primary sclerosing cholangitis and autoimmune hepatitis. There were 13 patients with multiple etiologies. *Increased frequency of PASD positivity in NASH cases $(P<0.001$, Fisher's exact test).

Over the course of 3 years, 11\% (21/196) of all liver explants in the primary cohort removed for ESLD had PASD+ hepatocyte globules. All PASD+ cases were also immunohistochemically positive for A1AT. Of these, 20 (95.2\%) were strongly and diffusely reactive for A1AT, whereas a single weakly PASD+ case (4.8\%) was only focally positive. In general, the PASD/ $\mathrm{A} 1 \mathrm{AT} \mathrm{IHC}+$ globules tended to be more conspicuous in hepatocytes at the periphery of cirrhotic nodules (Fig. 2).

Among the 196 patients from all etiologies in the 3-year primary cohort (Fig. 1), PASD+ globules were identified significantly more often in explants performed for NASH than for other causes $(P<0.001)$ : $47 \%$ of NASH; $16 \%$ of chronic alcohol abuse; $6.7 \%$ of $\mathrm{HCV}$ chronic infection; and $6.6 \%$ of other etiologies ( 1 case of A1ATD, 2 cases of autoimmune hepatitis, and 1 case of primary sclerosing cholangitis). Within the secondary expanded NASH cohort $(\mathrm{n}=33)$, the overall rate of PASD+ globules was similarly increased $(42 \% ; 14 / 33)$. All PASD+ NASH cases were $\mathrm{A} 1 \mathrm{AT}+$, and all PASD- NASH cases were A1AT-.

The mean BMI for all patients at the time of the liver transplant in the primary cohort was $27 \mathrm{~kg} / \mathrm{m}^{2}$. The mean BMI in PASD+ patients was $32 \mathrm{~kg} / \mathrm{m}^{2}$, whereas the mean BMI in PASD- patients $\left(27 \mathrm{~kg} / \mathrm{m}^{2}\right)$ 

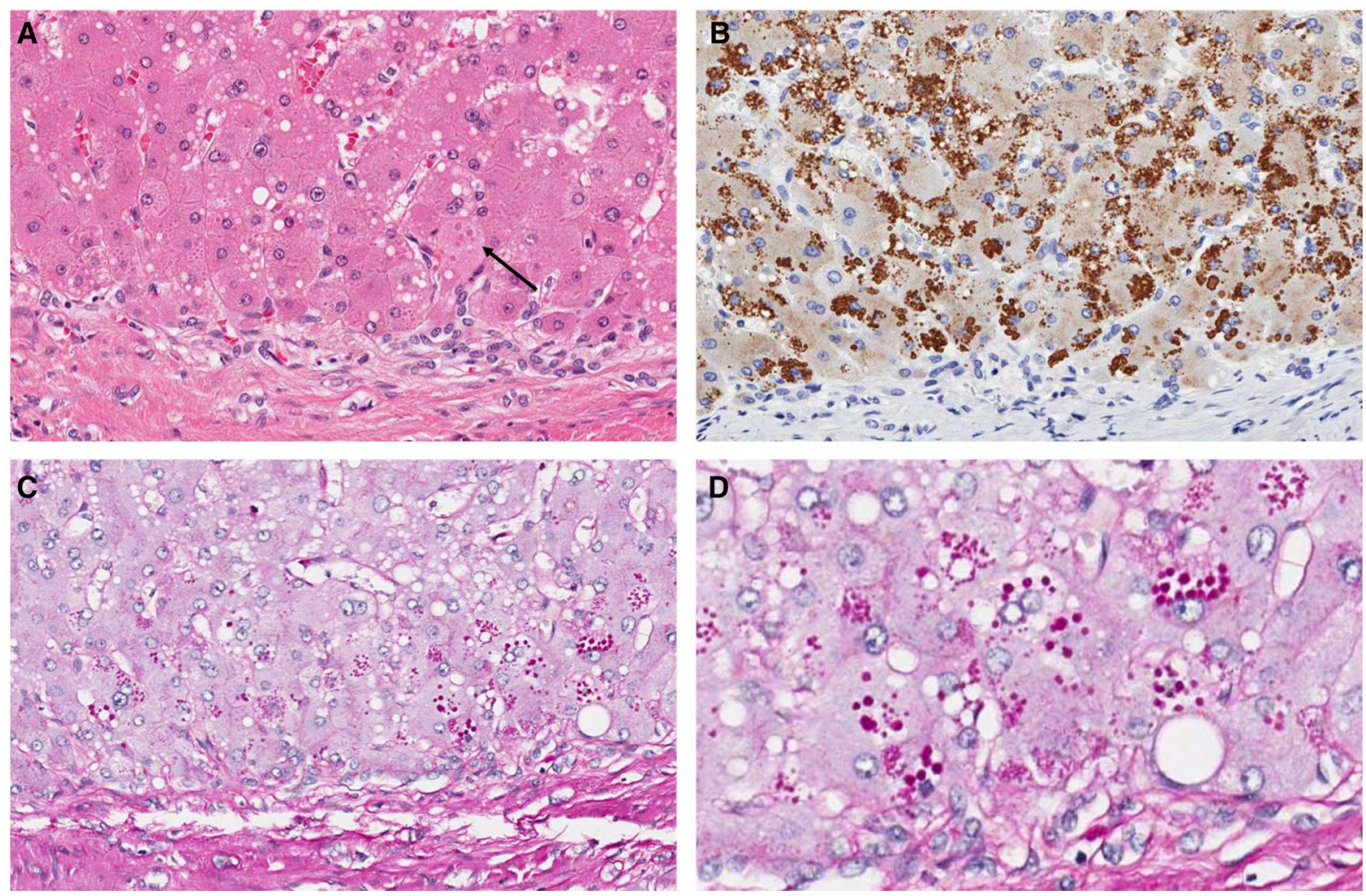

FIG. 2. (A) An example of subtle cytoplasmic eosinophilic globules (see arrow) within the cytoplasm of cirrhotic hepatocytes on standard hematoxylin-eosin staining. (B) The confirmatory A1AT immunohistochemical staining strongly highlights prominent A1AT protein positivity in the cirrhotic hepatocytes. (C) and (D) Globules are highlighted as a bright magenta color within the hepatocyte cytoplasm with PASD stain.

was significantly less $(P<0.001$; Table 1$)$. PASD+ $\mathrm{NASH}$ patients had a lower BMI on average (mean $\mathrm{BMI}, 31 \mathrm{~kg} / \mathrm{m}^{2}$ ) than PASD- NASH patients (mean BMI, $33 \mathrm{~kg} / \mathrm{m}^{2}$ ), although this difference was not statistically significant $(P=0.15)$. The increase in BMI in patients with PASD+ globules, however, may represent ascites because the majority of patients with PASD+ globules had ascites $(62 \% ; 13 / 21)$.

Of the patients with PASD+ globules at time of explant, only a single patient had a clinical workup for A1ATD. This was the primary driver for the development of an in-house RT-PCR-based assay to genotype the SERPINA1 gene from FFPE. A total of $85 \%$ of patients with strong PASD+ globules $(\mathrm{n}=26$, including those patients from the primary and secondary expanded NASH cohorts) were homozygous $(\mathrm{n}=2)$ or heterozygous $(\mathrm{n}=20)$ for the SERPINA1 $\mathrm{Z}$ allele. The 1 patient who was weakly PASD+ with only focal A1AT staining was negative for the SERPINA1 Z allele. The $\mathrm{S}$ allele was not detected in any PASD+
TABLE 1. Demographic and Clinical Data for Patients With Liver Explant Specimens Containing PASD+ and PASDHepatocyte Globules in the Primary Cohort

\begin{tabular}{lccc} 
& PASD $+(n=21)$ & PASD $-(n=175)$ & PValue \\
\hline Age, years & 54 & 56 & 0.64 \\
BMl, kg/m² & 32 & 27 & 0.001 \\
Sex, female & $9(43)$ & $54(31)$ & \\
Ethnicity & & & \\
$\quad$ Caucasian & $18(86)$ & $133(76)$ & \\
Asian & $0(0.0)$ & $13(7.4)$ & \\
Hispanic & $2(9.5)$ & $8(4.6)$ & \\
American Indian & $1(4.8)$ & $8(4.6)$ & \\
African American & $0(0.0)$ & $6(3.4)$ & \\
Middle Eastern & $0(0.0)$ & $4(2.3)$ & \\
Other & $0(0.0)$ & $3(1.7)$ &
\end{tabular}

NOTE: Data are given as n (\%) or mean.

globule patients (Table 2). Among the strong PASD+ patients, 1 sample failed quality control (QC) and was excluded in the analysis. In patients who underwent 
TABLE 2. $Z$ and S Allele Frequency Among Patients With Strong PASD+ Hepatocyte Globule Staining in Both the Primary and Expanded NASH Cohorts

\begin{tabular}{lc} 
& $\begin{array}{c}\text { Strong } \\
\text { PASD }+\end{array}$ \\
\hline Z or ZZ allele (QC pass) & $22 / 26(85)$ \\
S or SS allele (QC pass) & $0 / 26(0)$ \\
QC fail/total & $1 / 27(3.7)$
\end{tabular}

NOTE: Data are given as n (\%). A single patient sample failed QC on 2 repetitions of the assay and was therefore not included in the analysis of $\mathrm{Z}$ or $\mathrm{S}$ allele frequency.

TABLE 3. $Z$ and S Allele Frequency in NASH Patients With and Without PASD+ Hepatocyte Globule Staining

\begin{tabular}{lcc} 
& PASD + NASH & PASD - NASH \\
\hline Z or ZZ allele (QC pass) & $12 / 13(92)$ & $0 / 7(0)$ \\
S or SS allele (QC pass) & $0 / 13(0)$ & $1 / 7(14)$ \\
QC fail/total runs & $1 / 14(7.1)$ & $12 / 19(63)$
\end{tabular}

NOTE: Data are given as $\mathrm{n}(\%)$. A single PASD+ patient sample and 12 PASD- patient samples had 2 QC run failures and were therefore not included in the analysis of $\mathrm{Z}$ or $\mathrm{S}$ allele frequency.

liver transplantation for NASH, 92\% of patients with PASD+ globules were homozygous $(n=1)$ or heterozygous $(\mathrm{n}=11)$ for the $\mathrm{Z}$ allele. In contrast, among PASD- liver explants performed for NASH, no Z alleles were detected. The PASD- NASH patients had a high percentage of QC failure (12/19 patients). No Z allele was detected among the 7 PASD- NASH cases that passed QC or among the 12 PASD- NASH cases that failed QC. Only samples that produced a threshold cycle $(\mathrm{Ct})<32.0$ were considered acceptable for genotype determination and inclusion in the analyses. A single patient in the PASD- NASH group had a heterozygous $\mathrm{S}$ allele detected (Table 3).

The prevalence of $Z$ alleles in the primary cohort of all etiologies of ESLD was 8.2\% (16/196), corresponding to $76.2 \%(16 / 21)$ of PASD+ patients. Among the 16 patients with a $Z$ allele detected in all primary etiologies of the ESLD cohort, 1 was homozygous and 15 were heterozygous. Hepatocellular carcinoma was identified in the liver explant specimen in 2 of the 15 patients with $Z$ allele heterozygosity among the primary all etiologies of ESLD cohort.

\section{Discussion}

Unexpected PASD+ globules, in the context of advanced liver disease, are a specific finding that indicates the presence of a mutant A1AT allele. In our cohort, all but 1 PASD+ patients had strong and diffuse A1AT immunohistochemical staining, and $85 \%$ of PASD+ patients demonstrated at least 1 SERPIN1A1 $\mathrm{Z}$ allele on genotyping. Furthermore, explants performed for a clinical diagnosis of ESLD due to NASH were highly enriched for the SERPIN1A1 $\mathrm{Z}$ allele, suggesting a synergistic relationship between the $M Z$ genotype and advanced fibrosis in NASH. Only a single patient within our cohort had minimal clinical evaluation for A1ATD after PASD+ globules were reported in the explanted liver. This highlights a potential gap, at least in the study institution, in the follow-up of potentially important information for family members. Anecdotally, due to this study's demonstration that PASD+ globules are specific to a mutant A1AT genotype and the increased overall rate of SERPIN1A1 $\mathrm{Z}$ alleles in the liver transplantation population, the study institution has increased clinical workup following an unexpected PASD+ result and now evaluates A1AT phenotype on all patients starting an evaluation for liver transplantation.

The rate of unexpected PASD+ globules in the primary cohort in this study (11\%) closely matches a prior study in which $10 \%$ of ESLD patients had PASD+ globules. ${ }^{(6)}$ Worldwide, $3.7 \%$ of the population has a variant A1AT genotype, ${ }^{(2)}$ so therefore, the frequency of PASD+ globules in our ESLD cohort is nearly 3 times that of non-MM patients. This suggests that the presence of A1AT globules is not simply reflective of the expected polymorphic variants of A1AT present in the population but represents a possible association between ESLD and A1AT globules.

PCR testing in this study showed that PASD+ globules in the cirrhotic explants were highly correlated with the presence of at least $1 \mathrm{Z}$ allele; $85 \%$ of PASD+ patients were either homozygous or, more commonly, heterozygous for the $\mathrm{Z}$ allele. It is perhaps not surprising that A1AT globules accumulate in patients with heterozygosity for the SERPINA1 gene $Z$ allele because this mutation leads to A1AT protein misfolding and intrahepatic polymerization. ${ }^{(2)}$ Among adults presenting with chronic liver failure, prior studies have demonstrated a higher percentage of MZ heterozygotes $(8.4 \%)$ than expected by chance in the general population ( $2 \%$ of the population of North America carries a single $Z$ allele). ${ }^{(12)}$ In our retrospective study, at least $10 \%$ of all patients reviewed (including all patients during 2013-2015 and all NASH patients during 2006-2013) showed at least heterozygosity for 
the $Z$ allele. Recently, Schaefer et al. similarly found the prevalence of $\mathrm{MZ}$ in liver transplantation was 9.7\%, whereas the prevalence of MS was not significantly different than the general population. ${ }^{(10)}$ These data clearly demonstrate that liver transplantation patients are enriched for the $Z$ allele compared with the general population.

Furthermore, in this study, nearly $50 \%$ of patients with ESLD clinically attributed to NASH had PASD+ globules, and in all but 1 of these PASD+ cases the patient carried at least $1 \mathrm{Z}$ allele. Remarkably, nearly $40 \%$ of ESLD patients who carried a clinical diagnosis of NASH were at least heterozygous for the $\mathrm{Z}$ allele, far in excess of the predicted rate of $2 \%$ in the general population and the $8 \%-10 \%$ previously showed in advanced liver disease patients. ${ }^{(12)}$ Because our genotypic assay only tests for the $\mathrm{Z}$ and $\mathrm{S}$ alleles, it is possible that the few patients with PASD+ A1AT globules that did not contain a $Z$ allele may have a rare pathogenic SERPINA1 allele that results in the accumulation of A1AT protein, such as the $\mathrm{M}_{\text {malton }}$ SERPINA1 allele. $^{(2)}$

Previous epidemiology research has pointed to a synergistic effect between homozygous A1ATD and other factors in the progression to advanced liver disease. Bowlus et al. demonstrated obesity as a predisposing risk factor for advanced liver disease in the subset of homozygous A1ATD patients who develop cirrhosis, ${ }^{(8)}$ and Propst et al. showed that individuals with $\mathrm{ZZ}$ or $\mathrm{Z}$ genotypes who developed chronic liver disease had high rates of concurrent viral hepatitis. ${ }^{(7)}$ The role of $Z$ allele heterozygosity in chronic liver disease, and in NASH in particular, is more controversial. Schaefer et al. found $9.7 \%$ of orthotropic liver transplants in their cohort to be $\mathrm{MZ}$ and that $\mathrm{MZ}$ was associated with more advanced liver disease. ${ }^{(10)}$ Graziadei et al. found $8.2 \%$ of their transplant population in the late 1990s to be MZ; of this, $26.9 \%$ carried a diagnosis of cryptogenic cirrhosis. ${ }^{(12)}$ Valenti et al. showed that more non-MM genotype patients were diagnosed with nonalcoholic fatty liver disease (10.8\% compared with $3.5 \% \mathrm{MM}$ ), but ultimately, they did not find an association with liver damage. ${ }^{(9)}$ Czaja found that NASH patients had a greater frequency of the A1ATD phenotype than those with chronic hepatitis $\mathrm{C}$ infection (20\% versus 7\%). ${ }^{(17)}$ One additional study from Europe also reported that the presence of A1AT heterozygosity was significantly higher in NASH patients than the general population. ${ }^{(18)}$ Several other studies pointed out associations between A1AT heterozygosity and chronic liver disease, including in alcoholic and cryptogenic chronic liver disease. ${ }^{(19-23)}$ Not all studies, however, have shown an increase in the frequency of a heterozygous $\mathrm{Z}$ allele in chronic liver disease. ${ }^{(13)}$

The results from this study suggest a relationship between heterozygosity for the SERPIN1A1 $\mathrm{Z}$ allele and those conditions (primarily NASH, but to a lesser extent ethanol-associated liver damage) where PASD+ is overrepresented in our cohort. In A1AT heterozygotes, it is possible that parenchymal inflammation and injury induced by conditions, such as NASH, result in both the A1AT globule accumulation and suppression of disposal mechanisms, such as autophagy, that have been shown to be important in the cellular handling of mutant $Z$ protein. ${ }^{(24,25)}$ The function of A1AT in playing its anti-inflammatory role may be subsequently decreased. (26) This may allow the increased secretion of chemokines and cytokines, such as tumor necrosis factor and interleukin 6, that occur in obese patients to remain unchecked. ${ }^{(27)}$ These mediators along with the increased free fatty acids and other proteins associated with metabolic dysregulation may lead to liver injury, NASH, and a higher propensity for progression to cirrhosis. NASH is currently the second leading cause of liver disease among adults awaiting liver transplantation in the United States. With the advent of improved therapies for $\mathrm{HCV}$ infection, ${ }^{(28)}$ it is expected that ESLD patients with NASH will soon outnumber those with HCV in the United States. ${ }^{(29)}$ Currently, there is a paucity of reliable markers to predict the risk of progression to advanced liver disease in patients with NASH. The major risk factors predictive of disease progression are diabetes and obesity, but even these have not been described in all longitudinal studies. ${ }^{(30)}$ Given the increasing prevalence of $\mathrm{NASH}$, identification of additional biomarkers that independently predict the risk of progression to ESLD might provide improved prognostic information and suggest changes in management, such as smoking cessation and evaluation of family members.

Limitations of the current study include its retrospective nature, overlapping clinical etiologies of ESLD, and our inability to independently validate the underlying etiology of ESLD in many patients. For most patients, only the cirrhotic explanted liver was available for pathologic review. In addition, serologic testing for A1AT concentration and phenotype in advanced cirrhosis and following liver transplantation are not accurate, and in our cohort, they were mostly not available. DNA was successfully extracted from 
FFPE liver tissue stored up to 10 years after surgical removal. Although only a single PASD+ case failed RT-PCR amplification, there was a high rate of QC failure among PASD- NASH cases, which precluded melting curve analysis and genotype determination in the slight majority of PASD- NASH cases. The higher number of QC failures among PASD- NASH explant samples is likely due to the older age of these specimens compared with samples that passed $\mathrm{QC}(\mathrm{Ct}$ $<32.0)$. A majority of the PASD- samples (83\%, 10/12) that failed QC were over 5 years old. Recent work by Guyard et al. demonstrated that compared with DNA immediately extracted from FFPE tissue, archived samples stored for a mean of 5.4 years had a $53 \%$ lower DNA quantity and that only $11 \%$ of the original DNA could be amplified by RT-PCR. ${ }^{(31)}$ Although the QC failures could introduce bias, the fact that no $Z$ alleles were detected in the PASD- samples that had amplifiable DNA is reassuring. An additional limitation in this analysis is the lack of histologic and genotype data of the full cohort of liver explants performed for all etiologies of ESLD to reduce the potential for bias in the NASH-only subset analysis.

Although SERPINA1 $\mathrm{Z}$ allele carriers are generally not thought to have a discernable clinical phenotype, this study suggests an obvious candidate. Interestingly, Chu et al. suggested that liver disease in A1ATD might be better understood as an age-dependent degenerative disease. ${ }^{(14)}$ Our study identified a liver transplantation population enriched for the $Z$ allele, with a relatively low number of liver explants in $\mathrm{MZ}$ patients with hepatocellular carcinoma. Heterozygosity for the $\mathrm{Z}$ allele has been shown to increase the risk of hepatocellular carcinoma. ${ }^{(32-34)}$ However, more recent reports have questioned this relationship. ${ }^{(35)}$ Although our cohort suggests a phenotype of liver disease in $Z$ allele heterozygotes of more advanced liver fibrosis when combined with certain comorbidities (NASH), it did not show a large increase in hepatocellular carcinoma in the $\mathrm{Z}$ allele heterozygotes. Additional studies evaluating families who are $Z$ allele carriers may lend insight into subtler phenotypes of $\mathrm{MZ}$ patients.

A1ATD evaluation has been recommended in cases of unexplained liver disease ${ }^{(15)}$; however, our results suggest that A1ATD evaluation may be appropriate in all individuals with progressive chronic liver disease. Furthermore, our observation that almost no patient had clinical information or follow-up after the demonstration of PASD+ globules in explanted livers highlights the need for evaluation of the patient and family, even after liver transplantation. Family members of affected individuals are at a 50\% risk for inheriting the $\mathrm{Z}$ allele, and there may be a role for screening or early intervention to reduce the likelihood of ESLD in family members. At a minimum, the strong correlation between PASD+ globules and heterozygosity for A1ATD in liver explants warrants clinical follow-up.

\section{REFERENCES}

1) Bornhorst JA, Greene DN, Ashwood ER, Grenache DG. $\alpha 1$ Antitrypsin phenotypes and associated serum protein concentrations in a large clinical population. Chest 2013;143:1000-1008.

2) Stoller JK, Lacbawan F, Aboussouan LS. Alpha-1 antitrypsin deficiency. In: Adam MP, Ardinger HH, Pagon RA, et al., editors. GeneReviews. Seattle, WA: University of Washington; 1993-2017.

3) Fairbanks KD, Tavill AS. Liver disease in alpha 1-antitrypsin deficiency: a review. Am J Gastroenterol 2008;103:2136-2141.

4) Qizilbash A, Young-Pong O. Alpha 1 antitrypsin liver disease differential diagnosis of PAS-positive, diastase-resistant globules in liver cells. Am J Clin Pathol 1983;79:697-702.

5) Callea F, Fevery J, De Groote J, Desmet VJ. Detection of Pi Z phenotype individuals by alpha-1-antitrypsin (AAT) immunohistochemistry in paraffin-embedded liver tissue specimens. J Hepatol 1986;2:389-401.

6) Iezzoni JC, Gaffey MJ, Stacy EK, Normansell DE. Hepatocytic globules in end-stage hepatic disease: relationship to alpha1antitrypsin phenotype. Am J Clin Pathol 1997;107:692-697.

7) Propst T, Propst A, Dietze O, Judmaier G, Braunsteiner H, Vogel W. High prevalence of viral infection in adults with homozygous and heterozygous alpha 1-antitrypsin deficiency and chronic liver disease. Ann Intern Med 1992;117:641-645.

8) Bowlus CL, Willner I, Zern MA, Reuben A, Chen P, Holladay $\mathrm{B}$, et al. Factors associated with advanced liver disease in adults with alpha1-antitrypsin deficiency. Clin Gastroenterol Hepatol 2005;3:390-396.

9) Valenti L, Dongiovanni P, Piperno A, Fracanzani AL, Maggioni M, Rametta R, et al. Alpha 1-antitrypsin mutations in NAFLD: high prevalence and association with altered iron metabolism but not with liver damage. Hepatology 2006;44:857-864.

10) Schaefer B, Mandorfer M, Viveiros A, Finkenstedt A, Ferenci $P$, Schneeberger $\mathrm{S}$, et al. Heterozygosity for the alpha-1-antitrypsin $\mathrm{Z}$ allele in cirrhosis is associated with more advanced disease. Liver Transpl 2018;24:744-751.

11) Halangk J, Witt H, Puhl G, Gabelein G, Pascu M, Müller T, et al. Heterozygous alpha-1 antitrypsin deficiency as an inherited risk factor in the development of chronic liver disease. J Hepatol 2009;50:S162.

12) Graziadei IW, Joseph JJ, Wiesner RH, Therneau TM, Batts KP, Porayko MK. Increased risk of chronic liver failure in adults with heterozygous alpha1-antitrypsin deficiency. Hepatology 1998;28:1058-1063.

13) Regev A, Guaqueta C, Molina EG, Conrad A, Mishra V, Brantly $\mathrm{ML}$, et al. Does the heterozygous state of alpha- 1 antitrypsin deficiency have a role in chronic liver diseases? Interim results of a large case-control study. J Pediatr Gastroenterol Nutr 2006;43(suppl 1): S30-S35.

14) Chu AS, Chopra KB, Perlmutter DH. Is severe progressive liver disease caused by alpha-1-antitrypsin deficiency more common in children or adults? Liver Transpl 2016;22:886-894. 
15) Sandhaus RA, Turino G, Brantly ML, Campos M, Cross CE, Goodman $\mathrm{K}$, et al. The diagnosis and management of alpha-1 antitrypsin deficiency in the adult. Chronic Obstr Pulm Dis 2016;3:668-682.

16) Pac LJ, Cheeney G, Westerhoff $M$, Konnick EQ, Greene DN, Lockwood CM. Real-time PCR to detect $\alpha-1$ antitrypsin S and $Z$ Alleles in formalin-fixed paraffin-embedded tissue. J Appl Lab Med 2018;3:18-25.

17) Czaja AJ. Frequency and significance of phenotypes for alpha1antitrypsin deficiency in type 1 autoimmune hepatitis. Dig Dis Sci 1998;43:1725-1731.

18) Cacciottolo TM, Gelson WT, Maguire G, Davies SE, Griffiths WJ. Pi*Z heterozygous alpha- 1 antitrypsin states accelerate parenchymal but not biliary cirrhosis. Eur J Gastroenterol Hepatol 2014;26:412-417.

19) Fischer HP, Ortiz-Pallardó ME, Ko Y, Esch C, Zhou H. Chronic liver disease in heterozygous alpha1-antitrypsin deficiency $\mathrm{PiZ}$. J Hepatol 2000;33:883-892.

20) Goltz D, Hittetiya K, Vössing LM, Kirfel J, Spengler U, Fischer HP. $\mathrm{A}_{1}$-antitrypsin PiMZ heterozygosity has an independent aggravating effect on liver fibrosis in alcoholic liver disease. Virchows Arch 2014;465:539-546.

21) Bell H, Schrumpf E, Fagerhol MK. Heterozygous MZ alpha-1-antitrypsin deficiency in adults with chronic liver disease. Scand J Gastroenterol 1990;25:788-792.

22) Hodges JR, Millward-Sadler GH, Barbatis C, Wright R. Heterozygous $\mathrm{MZ}$ alpha 1-antitrypsin deficiency in adults with chronic active hepatitis and cryptogenic cirrhosis. N Engl J Med 1981;304:557-560.

23) Carlson J, Eriksson S. Chronic 'cryptogenic' liver disease and malignant hepatoma in intermediate alpha 1-antitrypsin deficiency identified by a $\mathrm{Pi} Z$-specific monoclonal antibody. Scand J Gastroenterol 1985;20:835-842.

24) Teckman JH. Emerging concepts and human trials in alpha1-antitrypsin deficiency liver disease. Semin Liver Dis 2017;37: $152-158$.
25) Perlmutter DH. Alpha-1-antitrypsin deficiency: importance of proteasomal and autophagic degradative pathways in disposal of liver disease-associated protein aggregates. Annu Rev Med 2011;62:333-345.

26) Stockley RA. The multiple facets of alpha-1-antitrypsin. Ann Transl Med 2015;3:130.

27) Papatheodoridi AM, Chrysavgis L, Koutsilieris M, Chatzigeorgiou A. The role of senescence in the development of non-alcoholic fatty liver disease and progression to non-alcoholic steatohepatitis. Hepatology 2019. https://doi.org/10.1002/hep. 30834.

28) Kohli A, Shaffer A, Sherman A, Kottilil S. Treatment of hepatitis C: a systematic review. JAMA 2014;312:631-640.

29) Wong RJ, Aguilar M, Cheung R, Perumpail RB, Harrison SA, Younossi ZM, Ahmed A. Nonalcoholic steatohepatitis is the second leading etiology of liver disease among adults awaiting liver transplantation in the United States. Gastroenterology 2015;148:547-555.

30) Calzadilla Bertot L, Adams LA. The natural course of nonalcoholic fatty liver disease. Int J Mol Sci 2016;17:E774.

31) Guyard A, Boyez A, Pujals A, Robe C, Tran Van Nhieu J, Allory $\mathrm{Y}$, et al. DNA degrades during storage in formalin fixed and paraffin-embedded tissue blocks. Virchows Arch 2017;471: 491-500.

32) Eriksson S, Carlson J, Velez R. Risk of cirrhosis and primary liver cancer in alpha 1-antitrypsin deficiency. N Engl J Med 1986;314:736-739.

33) Zhou H, Fischer HP. Liver carcinoma in PiZ alpha-1-antitrypsin deficiency. Am J Surg Pathol 1998;22:742-748.

34) Zhou H, Ortiz-Pallardó ME, Ko Y, Fischer HP. Is heterozygous alpha-1-antitrypsin deficiency type PIZ a risk factor for primary liver carcinoma? Cancer 2000;88:2668-2676.

35) Antoury C, Lopez R, Zein N, Stoller JK, Alkhouri N. Alpha-1 antitrypsin deficiency and the risk of hepatocellular carcinoma in end-stage liver disease. World J Hepatol 2015;7: $1427-1432$. 